\title{
Febrile Coronavirus and Autism
}

\author{
Gary Steinman* and David Mankuta
}

Department of Obstetrics and Gynecology, Hadassah Hospital, Hebrew University, Ein Kerem, Israel

\begin{abstract}
Background: A number of reports have pointed to the apparent relationship between reduced IGF1 in pregnancies and the development of autism in the offspring. Fever due to inflammatory processes in the gravida emerges as a potential key factor in this phenomenon.
\end{abstract}

Methods of inquiry: Investigations have reported an increase in neonatal autism related to an enhanced production of interleukins in some gravidas before delivery. The medical records of the mothers of autistic children were examined retrospectively for pertinent factors. Women who had endured infection- related fevers during their pregnancies were more likely to have delivered children with neurologic deficiencies such as autism and schizophrenia.

Conclusions: Inflammatory occurrences such as with women infected by Covid-19 during pregnancy produce enhanced levels of cytokines, especially interleukin-6, which can disrupt IGF1-mediated neurogenesis and CNS circuitry formation in the neonate.

\section{Keywords}

Autism, Coronavirus, Interleukin, IGF1, Cytokine, Myelin

\section{Introduction}

Retrospective studies have evaluated the recorded data of autistic youngsters in Denmark, Norway, and the United States born to women who had experienced febrile prenatal episodes, especially in the second trimester, compared to those with totally afebrile antepartum courses. Autism frequency increased about 1.4-fold in the offspring of gravidas who had been affected with antepartum fever related to various viral ailments. The highest rate of neonatal autism occurred when the mother experienced three febrile episodes during the same pregnancy. In one such retrospective study in the United States, the increased risk of autism was reduced when the mothers were treated with antipyretics. However, the possibility of brain toxicity side-effects may be associated with over-use of acetaminophen, as observed in test animals; ibuprofen is not recommended during pregnancy, especially in the third trimester [1-10].

Insulin-like growth factor-1 (IGF1) has numerous roles in the body's chemistry and homeostasis. Among these functions is the development of the components of the central and peripheral nervous systems, including brain growth, oligodendrocytes, and axonal myelin sheaths. Sufficient IGF1 in the neonate is essential for the proper introduction and integration of newly formed nerves, especially during the first postpartum year. This contributes to the development and persistence of functional brain neuronal patterns, and the avoidance of supratentorial neurologic defects (dysconnectivity) in the child, especially in the psychobehavioral pathways [11-14].

In viral-promoted maternal infections, the fetus while still in utero appears to be somewhat shielded. Although recent studies have not identified any major immediate detrimental effects on the fetus whose mother is battling severe Covid-19, the rise of interleukin IL- 6 in a "cytokine storm" governs the extent of corporal temperature elevation, which reduces the level of IGF1 in the neonate. Consequently, cerebroneural dysconnectivity may persist, thereby enhancing the chances of autism developing in the child $[11,12]$.

However, inflammation in pregnant mothers such as from coronaviruses has been linked to mental disorders in their offspring [13]. Postpartum neonatal complications such as autism, ADHD, depression, cerebral palsy, schizophrenia, poor cognition, or mental retardation may still be consequences of antepartum maternal inflammation. Interleukens such as IL6 may conceivably cross-over to the fetus, especially during the

*Corresponding author: Gary Steinman, Department of Obstetrics and Gynecology, Hadassah Hospital, Hebrew University, Ein Kerem, Jerusalem 12000, Israel

Accepted: December 28, 2021

Published online: December 30, 2021

Citation: Steinman G, Mankuta D (2021) Febrile Coronavirus and Autism. J Pediatr Neurol Neurosci 5(2):190-193 
middle months of the gestation. Excessive proinflammatory maternal cytokines reaching the fetal brain could be toxic to fetal neurons and oligodendrocytes in such instances [14].

There appears to be an inverse relationship between the level of maternal IL-6 during a pregnancy and the newborn's functional brain connectivity. Also, the formation of neonatal synapses may be altered by elevated IL- 6 in the mother and the consequential reduction of IGF1 in the fetus during the gestation. The diminution of IGF1 is central to subnormal myelination in the infant at basic stages of neural pathway development but may no longer be apparent in the older autistic patient [15]. Thus, a correlation exists between the degree of disturbance of the neonate's brain networks and the level of IL- 6 concentrations in the gravida. Similarly, an inverse association has been observed between front limbic cognitive development in the first 12 months of life of the neonate (or later) and the maternal IL- 6 concentration during the antecedent pregnancy. These are like the cognitive deficits commonly seen more clearly in autism. Persistence of neuroinflammation with IL-6 in the brains of mature autistic patients has been reported [12-14].

In related neurologic studies, behavior deviations found in children 2 years of age paralleled changes in maternal interleukin-6 levels during the prior gestation. In such cases, alterations in the mother's amygdala, in particular, preceded increased emotional and stress reactivity in their offspring [16-20].

Cytokine storm in the gravida, with fever and serious dyspnea, may follow a mild initial disease course. A surge in immune molecules, such as IL-6, and small blood clot production may result in fatal damage to the patient's lungs from acute respiratory distress syndrome (ARDS) $[11,21]$. This may well be the result of enhanced maternal hypoxemia and circulatory insufficiency.

\section{Pathophysiology of IL-6 vs. IGF-1}

Increased levels of maternal interleukins (especially IL6) have been measured in some samples of fetal blood and amniotic fluid [5,6]. Elevated IL-6 was detected postmortem in autistic brain tissue from affected individuals with an imbalance of neural circuitry. Maternal inflammation during the pregnancy, as measured by temperature and the IL- 6 level, appears to be associated with the newborn baby's reduced brain connectivity $[11,12]$. Overall, maternal fever for any infectious reason during gestation, especially within the middle months of the pregnancy, may enhance the potential for neonatal autism.

Typically, with an increase in IL-6, the level of IGF-1 falls. Especially in the newborn, IGF1 is the primary stimulant for myelination of new axonal fibers in the Central Nervous System. Patterson examined in detail the effect of glial and maternal-derived cytokine production in pregnancies. In particular, interleukin- 6 activated the placental JAK-STAT3 intermediate. As a result, the concentration of IGF-1 in the cerebrospinal fluid of neurologically normal youngsters was found to be higher than in autistic children of the same age [15-19].
Under stable biochemical conditions, the level of IGF1 often changes in the opposite direction from that of IL-6. For example, it has been reported that for babies born on or about the mean gestational age of 27.8 weeks, the average total serum level of IGF1 is $46.6 \mathrm{ng} / \mathrm{ml}$, whereas if the child is born at term without experiencing nutritional complications, the mean serum level is $88.7 \mathrm{ng} / \mathrm{ml}$. In contradistinction, the concentration of serum IL6 between these two gestational durations falls from 9.5 to $2.3 \mathrm{pg} / \mathrm{ml}(p<0.001)$ [20]. The physiologic states under which IL-6 or IGF-1 change dominates are central in defining the basis of various biomedical phenomena. For example, interleukin-6 increases in children with pathological inflammatory phenomena, cystic fibrosis, growth restriction bowel diseases, premature delivery due to maternal infection, and juvenile idiopathic arthritis. Similarly, chronic inflammatory states can be associated with IGF-1 insufficiency, IGF-1 resistance, or down-regulation of IGF receptors [21-27].

In recent years, infusion with rhIGF1 has been used to sustain and strengthen markedly premature babies born between 23 and 28 weeks of gestation. rhIGFBP-3 (IGF1 binding protein-3) is added to enhance and lengthen the duration of IGF1 support. This decreased the occurrence of severe bronchopulmonary dysplasia in the infants treated in this manner $[28,29]$.

On the one hand, IGF-1 enhancement is observed in mammals which resist febrile seizures [21]. Alternatively, growth-retarded neonates often present with higher concentrations of IL-6 in their placentas [15]. Local brain inflammation upsets the normal secretion of growth hormone from the baby's pituitary gland, resulting in IGF-1 insufficiency. Coronaviruses have been reported to invade neurons, causing demyelination $[30,31]$. Viral dissemination is possibly due to pathological changes in neurons which disturb brain circuits in particular [32].

It has been hypothesized that maternal immune activation (MIA), fever, and cytokine storm during pregnancy, associated with increased levels of IL-6, may be the etiologic disorder promoting neonatal neurologic ailments [12,33-36]. The exact role of IL-6, if exclusive, remains to be elucidated in the laboratory, but its relationship to IGF1 decrease may be the key to this febrile phenomenon.

The possible relevance of severe ARDS was again examined in June of this year. Five reports of IL6 elevation in Covid-19 cases in 2020 were compared with three prior reports (20142018) in other hyper inflammatory diseases. In the latter cases, the mean IL6 levels were 9 times or more higher than in the former cases. The analytical procedures were different in the former and latter sets [37].

However, this could intimate that inflammatory cytokines/ interleukin in addition to IL6, such as IL-17A, are operative in Covid-19. For example, the occurrence of cytokine storm in compromised individuals infected with Covid-19 is enhanced by chronic low-grade inflammation due to the combined effects of IL- $1 \beta$ L-6, IL-17A, and TNF- $\alpha$ (tumor necrosis factor) $[35,38]$. In autistic children, the more severe the autism, the higher are their serum IL-17A levels $[39,40]$. 
Brain dysconnectivity apparently due to defective myelination is a key finding in autism [41]. In test mice, decreased myelin-basic protein and other myelinrelated components have been found following influenza implantation [42]. This is especially worrisome in human babies experiencing long-term neurologic sequelae, as happened in Spanish flu.

\section{Prophylaxis}

It is concluded that positive action should be taken with all corona-exposed babies to compensate for any IGF1 deficiency, as measured in the umbilical cord blood at birth. Prior investigations have reported that infants who were nourished by breastfeeding exclusively for at least a full year had a lower incidence of autism subsequently. Human milk contains a higher level of IGF1 than milk from other sources. In addition, IGF1 survives gastric digestion in the baby, apparently due to the casein coating of the polypeptide [4345].

A study is now being initiated in cases of gravidas who survived severe Covid-19 during their pregnancies which examines the data related to the extent of breastfeeding the neonate and the degree of formation of autistic characteristics (if any) of the child at ages 1-2-years. This will be compared to mothers who were unaware of any febrile episodes during their pregnancies.

Hence, IGF-1 mediated myelination of developing CNS nerves in the neonate is central to understanding the effect of fever-promoted interleukins on patients affected by Covid- 19 . Such insight will be helpful if additional coronavirus-like pandemics occur in the future. Without being prepared for dealing with this, neuropediatric Ans will be challenged and short-staffed. In essentially all cases, febrile gravidas with flulike symptoms should be treated with antipyretics actively. Finally, the wide distribution of corona immunization, once successfully developed, would apparently result in a drop in autism rates in the offspring of pretreated women.

\section{References}

1. Atladottir HO, Thorsen P, Ostergaard L, et al. (2010) Maternal infection requiring hospitalization during pregnancy and autism spectrum disorders. J Autism Dev Disord 40: 1423-1430.

2. Zerbo P, Qian Y, Yoshida C, et al. (2015) Maternal infection during pregnancy and autism spectrum disorders. J Autism Dev Disord 45: 4015-4025.

3. Lee BK, Magnusson C, Gardner RM, et al. (2015) Maternal hospitalization with infection during pregnancy and risk of autism spectrum disorders. Brain Behav Immun 44: 100-105.

4. Fang SY, Wang S, Huang N, et al. (2015) Prenatal infection and autism spectrum disorders in childhood: A population-based case-control study in Taiwan. Paediatr Perinat Epidemiol 29: 307-316.

5. Hornig M, Bresnahan MA, Che X, et al. (2018) Prenatal fever and autism risk. Molec Psych 23: 759-766.

6. Abdallah MW, Larsen N, Grove J, et al. (2013) Amniotic fluid inflammatory cytokines: Potential markers of immunologic dysfunction in autism spectrum disorders. World J Biol Psychiatry
14: 528-538.

7. Atladottir HO, Henriksen TB, Schende DE, et al. (2012) Autism after infection, febrile episodes, and antibiotic use during pregnancy: An exploratory study. Pediatrics 130: e1447-e1454.

8. Zerbo O, losif AM, Walker C, et al. (2013) Is maternal influenza or fever during pregnancy associated with autism or developmental delays? Res from the Charge (Childhood Autism Risks from Genetics and environment) study. J Autism Dev Disorder 43: 25-33.

9. Croen LA, Qian Y, Ashwood P, et al. (2019) Infection and fever in pregnancy and autism spectrum disorders: findings from the study to explore early development. Autism Res 12: 1551-1556.

10. Viberg H, Eriksson P, Gordh T, et al. (2014) Paracetamol (acetaminophen) administration during neonatal brain development affects cognitive function and alters its analgesic and anxiolytic response in adult male mice. Toxicol Sci 138: 139-147.

11. Jonakait GM (2007) The effects of maternal inflammation on neuronal development: Possible mechanisms. Int J Devl Neurosci 25: 415-425.

12. Rudolph MD, Graham AM, Feczko, et al. (2018) Maternal IL-6 during pregnancy can be estimated from newborn brain connectivity andpredicts future working memory in offspring. Nat Neurosci 21: 765-777.

13. Wei H, Chadman KK, McCloskey DP, et al. (2012) Brain IL-6 elevation causes neuronal circuitry imbalances and mediates autism-like behaviors. Biochim Biophys Acta 1822: 831-842.

14. Rasmussen JM, Graham AM, Entringer S, et al. (2019) Maternal interleukin-6 concentration during pregnancy is associated with variation in frontolimbic white matter and cognitive development in early life. Neuroimag 185: 825-835.

15. Vanhala R, Turpeinen U, Riikonen R (2001) Low levels of insulinlike growth factor -1 in cerebrospinal fluid in children with autism. Dev Med Child Neurol 43: 614-616.

16. Patterson PH (2011) Maternal infection and immune involvement in autism. Trends Molec Med 17: 389-394.

17. Hsiao EY, McBride SW, Chow J, et al. (2012) Modeling an autism risk factor in mice leads to permanent immune dysregulation. PNAS 109: 12776-12781.

18. Hsiao EY, Patterson PH (2011) Activation of the maternal immune system induces endocrine changes in the placenta via IL-6. Brain Behav Immun 25: 604-615.

19. Tisoncik JR, Koreth MJ, Simmons CP, et al. (2012) Into the eye of the cytokinestorm. Microbiol Mol Biol Rev 76: 16-31.

20. Shmad I, Zaldivar F, Iwanaga K, et al. (2007) Inflammatory and growth mediators in growing preterm infants. J Ped Endoc Metab 20: 387-396.

21. Succurro E, Hribal ML, Andreozzi F, et al. (2008) Reciprocal association of plasma IGF-1 and interleukin- 6 levels with cardiometabolic risk factors in nondiabetic subjects. Diabetes Care 31: 1886-1888.

22. Sukhanov S, Higashi Y, Shai SY, et al. (2007) IGF-1 reduces inflammatory responses, suppresses oxidative stress, and decreases atherosclerosis progression in ApoE-deficient mice. Arterio Thromb Vasc Biol 27: 2684-2690

23. Street ME, Sehinin P, Fieni S, et al. (2006) Changes in interleukin-6 and IGF system and their relationships in placenta and cord blood in newborns with fetal growth restriction compared with controls. Eur J Endocrinol 155: 567-574. 
24. Cirillo F, Lazzeroni P, Sartori C, et al. (2017) Inflammatory diseases and growth: Effects on the GH-IGF axis and on growth plate. Int J Molec Sci 18: 1878.

25. Martins-Filho PR, Tanajura DM (2020) Covid-19 during pregnancy: Potential risk for neurodevelopmental disorders in neonates. Eur J Obs Gyn Reprod Biol 250: 255-256.

26. Steinman G, Mankuta D (2019) Molecular biology of autism's etiology - an alternative mechanism. Med Hypoth 130: 109272.

27. Koul O, Bauman M, Kemper TL, et al. (2005) The neurobiology of autism. ( $2^{\text {nd }}$ edn), Johns Hopkins, Maryland.

28. Ley D, Halberg B, Hansen-Pupp I, et al. (2019) rHIGF-1/rhIGFBP-3 in preterm infants: A phase 2 randomized controlled trial. J Ped 206: 56-65.

29. Hellstrom A, Ley D, Hallberg B, et al. (2017) IGF-1 as a drug for preterm intants: A step-wise clinical development. Cur Pharm Design 23: 5964-5970.

30. Wei H, Alberts I, Li X (2013) Brain IL-6 and autism. Neurosci 252: 320-325.

31. Graham AM, Rassmussen JM, Rudolph MD, et al. (2018) Maternal systemic interleukin- 6 during pregnancy is associated with newborn amygdala phenotypes and subsequent behavior at 2-years-of-age. Bio Psychiatry 83: 109-119.

32. Wegiel J, Flory M, Kuchna I, et al. (2014) Stereological study of the neuronal number and volume of 38 brain subdivisions of subjects diagnosed with autism reveals significant alterations restricted to the striatum, amygdala and cerebellum. Acta Neuropathol Commun 2: 141-147.

33. Masi A, Glozier N, Dale R, et al. (2017) The immune system, cytokines, and biomarkers in autism spectrum disorder. Neurosci Bull 33: 194-204.

34. Jiang H, Lian-lian X, Shao L, et al. (2016) Maternal infection during pregnancy and risk of autism spectrum disorders: A systematic review and meta-analysis. Brain Behav Immun 58: 165-172.
35. Boksa $P$ (2010) Effects of prenatal infection on brain development and behavior: A review of findings from animal models. Brain Behav Immun 24: 881-897.

36. Vargas DL, Nascimbene C, Krishnan C, et al. (2005) Neurological activation and neuroinflammation in the brain of patients with autism. Ann Neurol 57: 67-81.

37. Sinha P, Matthay MA, Calfee CS (2020) Is a "cytochrome storm" relevant to covid-19? JAMA Internal Medicine.

38. Leija-Martinez JJ, Huang F, Del-Rio-Navarro BE, et al. (2020) IL17A and TNF-a as potential biomarkers for acute respiratory distress syndrome and mortality in patients with obesity and Covid-19. Med Hypoth 114: 109935.

39. AL-Ayadhi LY, Mostafa GA (2012) Elevated serum levels of interleukin-17A in children with autism. J Neuroinflam 9: 158.

40. Raucci F, Mansour AA, Casillo GM, et al. (2020) Interleukin-17A (IL-17A), a key molecule of innate and adaptive immunity and its potential involvement in Covid-19-related thrombotic and vascular mechanisms. Autoimmunity Rev 19: 102572.

41. Steinman G (2015) Plausible etiology of brain dysconnectivity in autism - review and prospectus. Med Hypoth 85: 405-407.

42. Fatemi SH, Folsom TD, Reutiman TJ, et al. (2009) Abnormal expression of myelination genes and alterations in white matter fractional anisotropy following pre3natal influenza infection at E16 in mice. Schizophr Res 112: 46-53.

43. Steinman G, Mankuta D (2013) Breastfeeding as a possible deterrent to autism - A clinical perspective. Med Hypoth 81: 999-1001.

44. Steinman G (2020) Covid-19 and autism Med Hypoth 142: 109797.

45. Tseng PT, Chen YW, Stubbs D (2017) Maternal breastfeeding and autism spectrum disorder in children: A systematic review \& meta-analysis. Nutr Neurosci 18: 1-9.

DOI: $10.36959 / 595 / 436$

Copyright: (c) 2021 Steinman G, et al. This is an open-access article distributed under the terms of the Creative Commons Attribution License, which permits unrestricted use, distribution, and reproduction in any medium, provided the original author and source are credited. 